\title{
Strengthening the de Sitter swampland conjecture in warm inflation
}

\author{
Robert Brandenberger, ${ }^{a}$ Vahid Kamali ${ }^{a, b, c}$ and Rudnei O. Ramos ${ }^{d}$ \\ ${ }^{a}$ Department of Physics, McGill University, \\ Montreal, QC H3A 2T8, Canada \\ ${ }^{b}$ Department of Physics, Bu-Ali Sina (Avicenna) University, \\ Hamedan 65178 016016, Iran \\ ${ }^{c}$ School of Physics, Institute for Research in Fundamental Sciences (IPM), \\ Tehran 19538-33511, Iran \\ ${ }^{d}$ Departamento de Fisica Teorica, Universidade do Estado do Rio de Janeiro, \\ Rio de Janeiro, RJ 20550-013, Brazil \\ E-mail: rhb@hep.physics.mcgill.ca, vkamali@basu.ac.ir, rudnei@uerj.br
}

ABSTRaCT: The de Sitter constraint on the space of effective scalar field theories consistent with superstring theory provides a lower bound on the slope of the potential of a scalar field which dominates the evolution of the Universe, e.g., a hypothetical inflaton field. Whereas models of single scalar field inflation with a canonically normalized field do not obey this constraint, it has been claimed recently in the literature that models of warm inflation can be made compatible with it in the case of large dissipation. The de Sitter constraint is known to be derived from entropy considerations. Since warm inflation necessary involves entropy production, it becomes necessary to determine how this entropy production will affect the constraints imposed by the swampland conditions. Here, we generalize these entropy considerations to the case of warm inflation and show that the condition on the slope of the potential remains essentially unchanged and is, hence, robust even in the warm inflation dynamics. We are then able to conclude that models of warm inflation indeed can be made consistent with the swampland criteria.

KEywords: Cosmology of Theories beyond the SM, Spacetime Singularities

ARXiv EPrint: 2002.04925 


\section{Contents}

1 Introduction 1

2 Swampland conjectures through entropy considerations 2

3 Warm inflation and de Sitter conjecture 4

4 Examples $\quad 8$

4.1 Monomials chaotic inflation models 8

4.2 Hilltop type of inflation models 9

4.3 Generalized exponential type of inflation models 9

5 Conclusions $\quad 10$

\section{Introduction}

In order to understand the early evolution of the Universe at the highest energy scales, a consistent embedding of the physical model of the early Universe in a quantum theory of gravity is required. The only currently known consistent quantum gravity theory which unifies all forces of nature is superstring theory. Superstring theory leads to tight constraints (the so-called swampland constraints) on the space of possible effective field theories (EFT) which can be applied to study the evolution of the Universe. In particular, scalar fields arising in an EFT have a string-theoretical origin, and the range of validity of such EFTs and the potentials for the scalar fields are constrained. At the moment, the constraints are not at the level of theorems, but at the level of conjectures. The first conjecture (field range conjecture) [1] states that the field range over which an effective field theory for a canonically normalized scalar field $\phi$ is valid is bounded from above by a constant of order one times $M_{\mathrm{Pl}}$, the four-dimensional (reduced) Planck mass. The second condition (de Sitter conjecture) on the EFT of a scalar field $\phi$ which is introduced to explain the accelerated evolution of the early (or late) Universe [2] is that its potential $V(\phi)$ has a relative slope which is large in Planck units. ${ }^{1}$

These conditions are opposite to the conditions required to obtain successful cold ${ }^{2}$ slowroll inflation, at least in models of single field inflation based on a canonically normalized scalar field (the inflaton field) [7], but they remain consistent with quintessence models of Dark Energy [8]. On the other hand, in warm inflation (WI) models [9] (for reviews,

\footnotetext{
${ }^{1}$ See, for instance refs. [3, 4] for reviews, and ref. [5] for an understanding of the derivation of these conditions in the context of String Gas Cosmology [6].

${ }^{2}$ We speak of cold inflation (CI) if the damping term in the scalar field equation of motion is dominated by Hubble damping.
} 
see, e.g., refs. $[10,11])$ both the distance and de Sitter conjectures can be satisfied, as was discussed recently in refs. [12-22] in the context of high-dissipative models of WI.

In ref. [23], a derivation of the de Sitter conjecture was proposed which combines the distance criterion with an entropy argument. It is postulated that the entropy within a Hubble volume due to low mass bulk states cannot become larger than the Gibbons-Hawking entropy [24]. This is Bousso's [25] covariant entropy bound applied to a cosmological background. In the case of WI, there is an extra source of bulk energy, namely the thermal states into which part of the inflaton energy density is converted to through dissipative effects. Dissipation effects during slow-roll WI lead to a thermal bath of light particles which are interacting with the inflaton $[10,11]$. In this work, we will study the modification of the form of the de Sitter conjecture by adding the extra entropy production during a period of WI. Since this entropy production can potentially lead to a tighter constraint on the slope of the potential, it needs to be carefully studied in order to determine whether the Bousso bound can still remain satisfied. We address this question and show that taking the additional bulk entropy into account does not lead to an important change in the condition on the slope of the potential and that, hence, WI remains compatible with the swampland conjectures. $^{3}$ The present work then provides a demonstration of the robustness of WI in addressing the swampland conjectures in the context of entropy considerations.

The paper is organized as follows. In section 2, we briefly review the swampland conjectures and, in particular, the de Sitter conjecture when addressed through entropy considerations. In section 3 we summarize the WI dynamics and discuss its application is the context of the present work. We explicitly show how the entropy production in WI changes the Bousso condition. In section 4, we discuss some general models of WI and different primordial inflaton potentials demonstrating the robustness of WI as far as its compatibility with the de Sitter conjecture is concerned. Finally, in section 5, we give our conclusions.

\section{Swampland conjectures through entropy considerations}

In ref. [1] it was argued that the range of applicability of any low energy EFT which results after compactification to four spacetime dimensions of a ten-dimensional string theory is constrained. Specifically, the change $\triangle \phi$ of any scalar field arising in the EFT from its original point in field space obeys the upper bound

$$
\frac{\triangle \phi}{M_{\mathrm{Pl}}} \leq c_{1}
$$

\footnotetext{
${ }^{3}$ Note that the Trans-Planckian Censorship Conjecture (TCC), which has recently been proposed [26, 27] remains a challenge also for WI. This has also been addressed in $[17,18]$. The TCC, in the form proposed in $[26,27]$, demands that no sub-Planckian modes can ever exit the Hubble horizon, and this implies that for exponential inflation the number $N$ of e-foldings of inflation must be smaller than $\log \left(M_{\mathrm{Pl}} / H\right)$. Successful inflation is then only possible if $H / M_{\mathrm{P} 1}<10^{-20}$. A modified version of the TCC has recently been suggested in [28] (see also [29]) which posits that $N$ is bounded only by the logarithm of the de Sitter entropy, i.e. $N<2 \log \left(M_{\mathrm{Pl}} / H\right)$. In this case, somewhat larger values of $H$ during inflation (again assumed to be almost exponential) are possible, the bound becoming $H / M_{\mathrm{Pl}}<10^{-12}$.
} 
where $c_{1}$ is a constant of order one and $M_{\mathrm{Pl}} \equiv 1 / \sqrt{8 \pi G} \simeq 2.4 \times 10^{18} \mathrm{GeV}$ is the reduced Planck mass, defined in terms of the usual Newton gravitational constant $G$. Equation (2.1) is the distance conjecture. The reason for this bound is that if the scalar field moves a distance larger than that given by eq. (2.1), then a tower of new string states becomes low mass and must be included in the low energy EFT.

Later $[2,23,30]$, it was argued that the self-interaction potential of a scalar field which dominates the energy density of the Universe is constrained by one of the following conditions - either

$$
\frac{|\nabla V|}{V} \geq \frac{c_{2}}{M_{\mathrm{Pl}}}
$$

or

$$
\frac{\min \left(\nabla_{i} \nabla_{j} V\right)}{V} \leq-\frac{c_{3}}{M_{\mathrm{Pl}}^{2}},
$$

where $\nabla$ is the gradient in field space, $c_{2}$ and $c_{3}$ are universal and positive constants of order 1 and $\min \left(\nabla_{i} \nabla_{j} V\right)$ is the minimum eigenvalue of the Hessian $\nabla_{i} \nabla V_{j}$ in an orthonormal frame. This means that either the potential is sufficiently steep, or else sufficiently tachyonic. The condition (2.3) is used near the maximum of the potential, if it has one. Note that these conditions on the potential are trivially satisfied for a non-positive potential, or for $M_{\mathrm{Pl}} \rightarrow \infty$ (see refs. [1, 7, 23]).

As shown in ref. [23], one can use entropy arguments to derive the first part of de Sitter conjecture, given by eq. (2.2), from the distance conjecture, eq. (2.1), in the weak coupling regime of string theory, where the physical observables are under control. To be specific, let us assume that $\phi$ is an increasing function of time. The distance conjecture states that as $\phi$ grows, there is an increasing number $N(\phi)$ of effective degrees of freedom which can be excited and which must enter into the low energy EFT. Specifically,

$$
N(\phi) \sim n(\phi) \exp \left(b \frac{\phi}{M_{\mathrm{Pl}}}\right)
$$

is the number of light particles with small masses,

$$
m \sim \exp (-a \triangle \phi)
$$

where $a$ and $b$ in the above equations are constant parameters that depend on the mass gap and other features of the tower of string states and $n(\phi)$ is a monotonic function of $\phi$ with

$$
\frac{d n(\phi)}{d \phi}>0
$$

In an accelerating Universe with Hubble horizon $R=1 / H$, the increasing number of effective degrees of freedom given by eq. (2.4) leads to an increasing entropy of the tower of states, which can be parametrized by

$$
S_{\text {tower }}(N, R)=N^{\gamma}\left(M_{\mathrm{Pl}} R\right)^{\delta},
$$


where $\gamma$ and $\delta$ are constants. For example, if the new degrees of freedom behave as point particles, then $\delta=0$. In the accelerating Universe the entropy (2.7) is bounded by the Gibbons-Hawking entropy as [25]

$$
N^{\gamma}\left(M_{\mathrm{Pl}} R\right)^{\delta} \leq 8 \pi^{2} R^{2} M_{p}^{2} .
$$

Assuming that the potential energy dominates the total energy content, we can express the Hubble rate in terms of the potential energy density and rewrite eq. (2.8) as

$$
\frac{V}{3 M_{\mathrm{Pl}}^{4}} \leq\left(\frac{8 \pi^{2}}{N^{\gamma}}\right)^{1 /(1-\delta / 2)} .
$$

By taking the logarithm of both sides of eq. (2.9) and then differentiating with respect to $\phi$, we obtain

$$
\frac{V^{\prime}}{V} \leq-\frac{\gamma}{1-\delta / 2}(\ln (N))^{\prime},
$$

where the prime in the above equation and the following ones denotes a derivative with respect to $\phi .{ }^{4}$ With our convention for the running of the field, $V^{\prime}$ is negative and, hence, eq. (2.10) reads

$$
\frac{\left|V^{\prime}\right|}{V} \geq \frac{\gamma}{1-\delta / 2}(\ln (N))^{\prime} .
$$

Using eqs. (2.4) and (2.6), we obtain

$$
\frac{\left|V^{\prime}\right|}{V} \geq \frac{2 \gamma}{2-\delta}\left(\frac{n^{\prime}}{n}+\frac{b}{M_{p}}\right)>\frac{1}{M_{p}} \frac{2 b \gamma}{2-\delta},
$$

where $\delta<2$ according to ref. [23]. The result given by eq. (2.12) can be compared to the swampland de Sitter condition eq. (2.2), where the constant $c_{2}$ is then identified to be given by

$$
c_{2}=\frac{2 b \gamma}{2-\delta} .
$$

In the following section we will extend the above analysis to WI.

\section{Warm inflation and de Sitter conjecture}

In the WI scenario, dissipative effects intrinsic to the interactions of the inflaton with other field degrees of freedom [10] can lead to a sustainable radiation production throughout the inflationary expansion. Those interactions with other field degrees of freedom, for some region of parameters, can be nonnegligible, generating dissipation terms and converting a small fraction of vacuum energy density into radiation. When the magnitude of these dissipation terms is strong enough to compensate the redshift of the radiation by the expansion, a steady state can be produced, with the inflationary phase happening in a thermalized radiation bath. The background inflaton dynamics then becomes

$$
\ddot{\phi}+(3 H+\Upsilon) \dot{\phi}+V^{\prime}=0,
$$

\footnotetext{
${ }^{4}$ Strictly speaking, this conclusion is valid provided that we consider situations when the inequality $(2.9)$ is close to being saturated.
} 
while the evolution equation for the radiation fluid energy density $\rho_{r}$ is

$$
\dot{\rho}_{r}+4 H \rho_{r}=\Upsilon \dot{\phi}^{2},
$$

where $\Upsilon$ denotes the dissipative coefficient in WI and $H$ is the Hubble parameter as usual. The entropy density $s$ is related to the radiation energy density by $T s=\left(1+w_{r}\right) \rho_{r}$, where $w_{r}$ is the thermal bath equation of state. Then, eq. (3.2) can be written in terms of the entropy density as

$$
T \dot{s}+3 H T s=\Upsilon \dot{\phi}^{2},
$$

where we have considered a thermalized radiation bath, i.e., $w_{r}=1 / 3$, as is typically the case in WI, where then

$$
\rho_{r}=\alpha T^{4}
$$

where $\alpha=\pi^{2} g_{*} / 30$ and $g_{*}$ is the effective number of radiation degrees of freedom, while the associated entropy density is

$$
s=\frac{4}{3} \alpha T^{3} .
$$

During the slow-roll epoch, eqs. (3.1), (3.2) and (3.3) become, respectively,

$$
\begin{aligned}
3 H(1+Q) \dot{\phi} & \simeq-V^{\prime}, \\
\rho_{r} & \simeq \frac{3 Q}{4} \dot{\phi}^{2}, \\
T s & \simeq Q \dot{\phi}^{2},
\end{aligned}
$$

where $Q$ denotes the dissipation rate in WI, defined as

$$
Q \equiv \frac{\Upsilon}{3 H},
$$

while for the Hubble parameter, since during WI we have that $V \gg \rho_{r}$, we have

$$
H^{2} \simeq \frac{V}{3 M_{\mathrm{Pl}}^{2}}
$$

Combining eqs. (3.6) and (3.7) and also using eq. (3.4), we obtain

$$
\alpha T^{4} \simeq \frac{\left(V^{\prime}\right)^{2}}{12 H^{2}} \frac{Q}{(1+Q)^{2}}
$$

We can add the entropy of the thermal bath to the left-hand side of eq. (2.8). Demanding that the Bousso bound remains satisfied, we are then led to the condition

$$
N^{\gamma}\left(M_{\mathrm{Pl}} R\right)^{\delta}+\frac{4}{3} \pi s R^{3} \leq 8 \pi^{2}\left(M_{\mathrm{Pl}} R\right)^{2},
$$

which implies that $N$ is bounded by

$$
N^{\gamma} M_{\mathrm{Pl}}^{\delta} \leq R^{3-\delta}\left(8 \pi^{2} R^{-1} M_{\mathrm{Pl}}^{2}-\frac{4}{3} \pi s\right) .
$$


Assuming that the potential energy density dominates the total energy density, it then allows us to substitute for $R=1 / H$ making use of the Friedmann equation in the slow-roll approximation, eq. (3.10), to obtain

$$
R=\sqrt{3} \frac{M_{\mathrm{Pl}}}{\sqrt{V}}
$$

Making use of eq. (2.4), taking the logarithm of both sides of eq. (3.13) and differentiating with respect to the scalar field, we obtain

$$
\gamma\left(\frac{b}{M_{\mathrm{Pl}}}+\frac{n^{\prime}}{n}\right) \leq-\frac{3-\delta}{2} \frac{V^{\prime}}{V} \times\left(1-\frac{1}{3-\delta} \frac{1-\frac{\dot{s}}{6 \pi M_{\mathrm{P}}^{2} \dot{H}}}{1-\frac{s}{6 \pi M_{\mathrm{Pl}}^{2} H}}\right),
$$

where we have made use of the relation

$$
\frac{4 \pi}{3} s^{\prime} \simeq \frac{2 \pi}{3} \frac{V^{\prime}}{V} H \frac{\dot{s}}{\dot{H}} .
$$

Comparing eq. (3.15) to eq. (2.12), we have that the WI modification to the de Sitter swampland conjecture can be expressed as

$$
\frac{\left|V^{\prime}\right|}{V}>\tilde{c}_{2}
$$

where $\tilde{c}_{2}$ is given by

$$
\tilde{c}_{2}=\frac{2 \gamma b}{3-\delta}\left|1-\frac{1}{3-\delta} \frac{1-\frac{\dot{s}}{6 \pi M_{\mathrm{Pl}}^{2} \dot{H}}}{1+\frac{s}{6 \pi M_{\mathrm{Pl}}^{2} H}}\right|^{-1} .
$$

To determine the magnitude of the WI modification in eq. (3.18), we need then to estimate values for the ratios $s / H$ and $\dot{s} / \dot{H}$. First, note that we can write the ratio $s / H$ as

$$
\frac{s}{H} \simeq \frac{Q \dot{\phi}^{2}}{T H} \simeq \frac{Q}{1+Q} 2 M_{\mathrm{Pl}}^{2} \frac{H}{T} \frac{\epsilon_{\phi}}{1+Q} \simeq \frac{Q}{1+Q} 2 M_{\mathrm{Pl}}^{2} \frac{H}{T} \epsilon_{H},
$$

where we have used the slow-roll equations (3.6), (3.8), (3.10) and also that

$$
\epsilon_{\phi}=\frac{M_{\mathrm{Pl}}^{2}}{2}\left(\frac{V^{\prime}}{V}\right)^{2}
$$

and that in WI,

$$
\epsilon_{H} \equiv-\frac{\dot{H}}{H^{2}}=\frac{\epsilon_{\phi}}{1+Q} .
$$

Since in WI we have by definition that $T>H$, thus, we conclude that the quantity $s /\left(6 \pi M_{\mathrm{Pl}}^{2} H\right)$ appearing in eq. (3.18) is much smaller than 1 ,

$$
\frac{s}{6 \pi M_{\mathrm{Pl}}^{2} H} \simeq \frac{1}{3 \pi} \frac{Q}{1+Q} \frac{H}{T} \epsilon_{H} \ll 1,
$$


which is valid in both the weak and strong dissipative regimes in WI, i.e., $Q \ll 1$ and $Q \gg 1$, respectively, and recalling that current observations [31] limit the slow-roll parameter to values $\epsilon_{H} \lesssim 10^{-2}$. We also note that the result (3.22) holds not only deep in the inflationary regime, but it also remains true through the end of inflation, when $\epsilon_{H} \rightarrow 1$. This is because typically the ratio $T / H$ increases towards the end of inflation, which is a common feature of the known models of WI $[32,33]$.

Next, we notice that the term $\dot{s} /\left(6 \pi M_{\mathrm{Pl}}^{2} \dot{H}\right)$ can also be expressed as

$$
\frac{\dot{s}}{6 \pi M_{\mathrm{Pl}}^{2} \dot{H}}=\frac{s}{6 \pi M_{\mathrm{Pl}}^{2} H} \frac{s^{\prime} H}{s H^{\prime}},
$$

and

$$
\frac{s^{\prime} H}{s H^{\prime}} \simeq 6 \frac{T^{\prime} V}{T V^{\prime}}
$$

Hence, eq. (3.18) becomes,

$$
\tilde{c}_{2} \simeq \frac{2 \gamma b}{3-\delta}\left|1-\frac{1}{3-\delta}\left[1+\kappa\left(1-6 \frac{T^{\prime} V}{T V^{\prime}}\right)+\mathcal{O}\left(\kappa^{2}\right)\right]\right|^{-1}
$$

where

$$
\kappa=\frac{s}{6 \pi M_{\mathrm{Pl}}^{2} H} .
$$

To study the change in the coefficient $\tilde{c}_{2}$ between the cases of CI and WI we can parametrize the dissipation coefficient $\Upsilon$ as follows,

$$
\Upsilon(T, \phi)=C T^{n} \phi^{p} M^{1-n-p},
$$

where $C$ is a dimensionless constant (that carries the details of the microscopic model used to derive the dissipation coefficient, e.g., the different coupling constants of the model), $M$ is a mass scale in the model and depends of its construction, while $n$ and $p$ are numerical powers, which can be either positive or negative numbers. The parametrization eq. (3.27) covers many different models of WI that have been proposed in the literature (see, e.g., ref. [17] and references therein). As an additional note, we should also point out that the dynamical stability of the background equations in WI requires that the power $n$ in the dissipation coefficient eq. (3.27) satisfies $-4<n<4$, which is valid in the weak $(Q \ll 1)$ and strong $(Q>1)$ dissipative regimes of WI [34-36].

From eqs. (3.11) and (3.27) we find that the functional relation between the temperature and the amplitude of the inflaton field can be expressed, in the high dissipative regime $Q>1$, as

$$
T(\phi) \simeq\left[\frac{\sqrt{3} M_{\mathrm{Pl}}}{4 \alpha} \frac{\left(V^{\prime}\right)^{2}}{C \phi^{p} V^{1 / 2} M^{1-n-p}}\right]^{\frac{1}{4+n}},
$$

and, consequently, we find

$$
\frac{T^{\prime}}{T}=\frac{1}{4+n}\left(2 \frac{V^{\prime \prime}}{V^{\prime}}-\frac{1}{2} \frac{V^{\prime}}{V}-\frac{p}{\phi}\right)
$$


Substituting eq. (3.29) in the ratio $T^{\prime} V /\left(T V^{\prime}\right)$ appearing in eq. (3.25), we find that

$$
\left.\frac{T^{\prime} V}{T V^{\prime}}\right|_{Q>1} \simeq \frac{1}{4+n}\left[2 \frac{V V^{\prime \prime}}{\left(V^{\prime}\right)^{2}}-\frac{p V}{\phi V^{\prime}}-\frac{1}{2}\right] .
$$

Likewise, working now in the low dissipative regime of WI, $Q \ll 1$, we find that

$$
T(\phi) \simeq\left[\frac{\sqrt{3} M_{\mathrm{Pl}}^{3}}{12 \alpha} \frac{C \phi^{p} M^{1-n-p}\left(V^{\prime}\right)^{2}}{V^{3 / 2}}\right]^{\frac{1}{4-n}},
$$

and

$$
\frac{T^{\prime}}{T}=\frac{1}{4-n}\left(2 \frac{V^{\prime \prime}}{V^{\prime}}-\frac{3}{2} \frac{V^{\prime}}{V}+\frac{p}{\phi}\right)
$$

which gives for the ratio $T^{\prime} V /\left(T V^{\prime}\right)$ the result

$$
\left.\frac{T^{\prime} V}{T V^{\prime}}\right|_{Q \ll 1} \simeq \frac{1}{4-n}\left[2 \frac{V V^{\prime \prime}}{\left(V^{\prime}\right)^{2}}+\frac{p V}{\phi V^{\prime}}-\frac{3}{2}\right]
$$

\section{Examples}

Using the results given by eqs. (3.30) and (3.33), we can find the form of $\tilde{c}_{2}$ once the primordial inflaton potential is given. Let us consider some representative models next as examples.

\subsection{Monomials chaotic inflation models}

Let us consider the case of monomial chaotic inflation models given by the primordial potential,

$$
V(\phi)=\frac{V_{0}}{2 k}\left(\frac{\phi}{M_{\mathrm{Pl}}}\right)^{2 k} .
$$

Cases with $k=1,2$ and 3 are known to satisfy the observational constraints in the WI scenario $[37,38]$.

Using eq. (4.1) in eqs. (3.30) and (3.33), we find, respectively,

$$
\left.\frac{T^{\prime} V}{T V^{\prime}}\right|_{Q>1}=\frac{1}{4+n}\left(\frac{3}{2}-\frac{p+2}{2 k}\right)
$$

and

$$
\left.\frac{T^{\prime} V}{T V^{\prime}}\right|_{Q \ll 1}=\frac{1}{4-n}\left(\frac{1}{2}+\frac{p-2}{2 k}\right) .
$$

We can consider in the above equations two previous well studied dissipation coefficients in WI and with a quartic inflaton potential $(k=2)$ : (a) A cubic in the temperature dissipation coefficient [39], $\Upsilon=C T^{3} / \phi^{2}$, for which we have $n=3$ and $p=-2$. This gives for eq. (4.2) the result 3/14, while for eq. (4.3) it gives $-1 / 2$; (b) A linear in the temperature dissipation coefficient [32], $\Upsilon=C T$, for which we have $n=1$ and $p=0$. This gives for eq. (4.2) the result $1 / 5$, while for eq. (4.3) it gives 0 . This remains true also for other more general dissipation coefficients, like the ones recently studied in refs. [38, 40]. In all these cases we find that the ratio $T^{\prime} V /\left(T V^{\prime}\right)$ is in general smaller than 1 . Thus, $\tilde{c}_{2} \simeq c_{2}$ is obtained for all these WI models. 


\subsection{Hilltop type of inflation models}

Hilltop type of inflaton models are described by a potential of the form,

$$
V(\phi)=V_{0}\left[1-\beta\left(\frac{\phi}{M_{\mathrm{Pl}}}\right)^{2 k}\right] .
$$

These type of potentials, representing small field like models of inflations, can also be shown to be compatible with the observations in WI [37], with the constant $\beta$ in eq. (4.4) in general satisfying $\beta \sim 10^{-2}$.

As in the previous example, we find now that

$$
\left.\frac{T^{\prime} V}{T V^{\prime}}\right|_{Q>1}=\frac{1}{4+n}\left\{\frac{[p-2(2 k-1)]\left[1-\beta\left(\frac{\phi}{M_{\mathrm{Pl}}}\right)^{2 k}\right]}{2 \beta k\left(\frac{\phi}{M_{\mathrm{Pl}}}\right)^{2 k}}-\frac{1}{2}\right\},
$$

and

$$
\left.\frac{T^{\prime} V}{T V^{\prime}}\right|_{Q \ll 1}=-\frac{1}{4-n}\left\{\frac{[p+2(2 k-1)]\left[1-\beta\left(\frac{\phi}{M_{\mathrm{Pl}}}\right)^{2 k}\right]}{2 \beta k\left(\frac{\phi}{M_{\mathrm{Pl}}}\right)^{2 k}}+\frac{3}{2}\right\} .
$$

In the example studied for instance in ref. [37], where $k=1$ and $\beta \sim 10^{-2}$, we typically have $\phi>4 M_{\mathrm{Pl}}$ and ultraviolet completions for the potential (4.4) are also expected in general to be in a scale $m_{\mathrm{UV}} \gg M_{\mathrm{Pl}}$ when the potential is no longer unbounded. Considering also the most common dissipation terms used in the literature for WI, we expect that both eqs. (4.5) and (4.6) to be not larger than order 1. Thus, here also we can predict that $\tilde{c}_{2} \simeq c_{2}$.

\subsection{Generalized exponential type of inflation models}

Inflation models of a generalized exponential form given by

$$
V(\phi)=V_{0} e^{-\beta\left(\frac{\phi}{M_{\mathrm{Pl}}}\right)^{k}}
$$

have also been studied recently in the literature and found regions of parameter space compatible with the observations in the WI scenario, at least for cases with $k>2$ (see, e.g., ref. [41]).

As in the previous examples, we now have that

$$
\left.\frac{T^{\prime} V}{T V^{\prime}}\right|_{Q>1}=\frac{1}{4+n}\left[\frac{p-2(k-1)}{\beta k\left(\frac{\phi}{M_{\mathrm{Pl}}}\right)^{k}}+\frac{3}{2}\right],
$$


and

$$
\left.\frac{T^{\prime} V}{T V^{\prime}}\right|_{Q \ll 1}=-\frac{1}{4-n}\left[\frac{p+2(k-1)}{\beta k\left(\frac{\phi}{M_{\mathrm{Pl}}}\right)^{k}}-\frac{1}{2}\right] .
$$

In the type of inflation models with the potential (4.7) a scaling regime is fast approached when $\beta\left(\phi / M_{\mathrm{Pl}}\right)^{k} \gtrsim 1$ after the inflationary regime. In this case, both eqs. (4.5) and (4.6) are found to be not larger than order 1. Even during inflation, at least for the examples studied in ref. [41], we find that the product $\kappa\left[1-6 T^{\prime} V /\left(T V^{\prime}\right)\right]$ in eq. (3.25) is always found to be much smaller than 1 . Thus, here we also find $\tilde{c}_{2} \simeq c_{2}$.

\section{Conclusions}

We have generalized the derivation of the de Sitter constraint on effective field theories consistent with quantum gravity to the case of WI. We have shown that the condition on the slope of the scalar field potential is changed in a minimal way due to the entropy effects inherent of the WI dynamics. We have studied general classes of dissipation terms currently found in the WI literature and which are able to lead to consistent observational quantities. Different broad classes of primordial inflaton potentials were also studied. For all these cases, we found that the WI correction to the de Sitter swampland condition is always negligible. Hence, the warm inflation scenario can indeed be made compatible with the swampland conditions and we have demonstrated here that this is a robust result.

\section{Acknowledgments}

The research at McGill is supported in part by funds from NSERC and from the Canada Research Chair program. The visit of VK to McGill has also been supported in part by the McGill Space Institute. R.O.R. is partially supported by research grants from Conselho Nacional de Desenvolvimento Científico e Tecnológico (CNPq), Grant No. 302545/2017-4, and Fundação Carlos Chagas Filho de Amparo à Pesquisa do Estado do Rio de Janeiro (FAPERJ), Grant No. E-26/202.892/2017.

Open Access. This article is distributed under the terms of the Creative Commons Attribution License (CC-BY 4.0), which permits any use, distribution and reproduction in any medium, provided the original author(s) and source are credited.

\section{References}

[1] H. Ooguri and C. Vafa, On the Geometry of the String Landscape and the Swampland, Nucl. Phys. B 766 (2007) 21 [hep-th/0605264] [INSPIRE].

[2] G. Obied, H. Ooguri, L. Spodyneiko and C. Vafa, de Sitter Space and the Swampland, arXiv: 1806.08362 [INSPIRE].

[3] T.D. Brennan, F. Carta and C. Vafa, The String Landscape, the Swampland, and the Missing Corner, PoS TASI2017 (2017) 015 [arXiv:1711.00864] [INSPIRE]. 
[4] E. Palti, The Swampland: Introduction and Review, Fortsch. Phys. 67 (2019) 1900037 [arXiv: 1903.06239] [INSPIRE].

[5] S. Laliberte and R. Brandenberger, String Gases and the Swampland, JCAP 07 (2020) 046 [arXiv:1911.00199] [INSPIRE].

[6] R. Brandenberger and C. Vafa, Superstrings in the Early Universe, Nucl. Phys. B 316 (1989) 391 [INSPIRE].

[7] P. Agrawal, G. Obied, P.J. Steinhardt and C. Vafa, On the Cosmological Implications of the String Swampland, Phys. Lett. B 784 (2018) 271 [arXiv: 1806.09718] [INSPIRE].

[8] L. Heisenberg, M. Bartelmann, R. Brandenberger and A. Refregier, Dark Energy in the Swampland, Phys. Rev. D 98 (2018) 123502 [arXiv:1808.02877] [INSPIRE].

[9] A. Berera, Warm inflation, Phys. Rev. Lett. 75 (1995) 3218 [astro-ph/9509049] [InSPIRE].

[10] A. Berera, I.G. Moss and R.O. Ramos, Warm Inflation and its Microphysical Basis, Rept. Prog. Phys. 72 (2009) 026901 [arXiv:0808.1855] [InSPIRE].

[11] M. Bastero-Gil and A. Berera, Warm inflation model building, Int. J. Mod. Phys. A 24 (2009) 2207 [arXiv: 0902.0521] [InSPIRE].

[12] S. Das, Note on single-field inflation and the swampland criteria, Phys. Rev. D 99 (2019) 083510 [arXiv: 1809.03962] [INSPIRE].

[13] M. Motaharfar, V. Kamali and R.O. Ramos, Warm inflation as a way out of the swampland, Phys. Rev. D 99 (2019) 063513 [arXiv:1810.02816] [INSPIRE].

[14] S. Das, Distance, de Sitter and Trans-Planckian Censorship conjectures: the status quo of Warm Inflation, Phys. Dark Univ. 27 (2020) 100432 [arXiv:1910.02147] [InSPIRE].

[15] S. Das, Warm Inflation in the light of Swampland Criteria, Phys. Rev. D 99 (2019) 063514 [arXiv: 1810.05038] [INSPIRE].

[16] M. Bastero-Gil, A. Berera, R. Hernández-Jiménez and J.G. Rosa, Warm inflation within a supersymmetric distributed mass model, Phys. Rev. D 99 (2019) 103520 [arXiv:1812.07296] [INSPIRE].

[17] V. Kamali, M. Motaharfar and R.O. Ramos, Warm brane inflation with an exponential potential: a consistent realization away from the swampland, Phys. Rev. D 101 (2020) 023535 [arXiv: 1910.06796] [INSPIRE].

[18] A. Berera and J.R. Calderón, Trans-Planckian censorship and other swampland bothers addressed in warm inflation, Phys. Rev. D 100 (2019) 123530 [arXiv:1910.10516] [INSPIRE].

[19] V. Kamali, Non-minimal Higgs inflation in the context of warm scenario in the light of Planck data, Eur. Phys. J. C 78 (2018) 975 [arXiv:1811.10905] [InSPIRE].

[20] V. Kamali, Warm pseudoscalar inflation, Phys. Rev. D 100 (2019) 043520 [arXiv: 1901.01897] [INSPIRE].

[21] V. Kamali, Reheating After Swampland Conjecture, JHEP 01 (2020) 092 [arXiv: 1902.00701] [INSPIRE].

[22] S. Das, G. Goswami and C. Krishnan, Swampland, axions, and minimal warm inflation, Phys. Rev. D 101 (2020) 103529 [arXiv:1911.00323] [inSPIRE].

[23] H. Ooguri, E. Palti, G. Shiu and C. Vafa, Distance and de Sitter Conjectures on the Swampland, Phys. Lett. B 788 (2019) 180 [arXiv:1810.05506] [INSPIRE]. 
[24] G.W. Gibbons and S.W. Hawking, Cosmological Event Horizons, Thermodynamics, and Particle Creation, Phys. Rev. D 15 (1977) 2738 [InSPIRE].

[25] R. Bousso, A Covariant entropy conjecture, JHEP 07 (1999) 004 [hep-th/9905177] [INSPIRE].

[26] A. Bedroya and C. Vafa, Trans-Planckian Censorship and the Swampland, arXiv: 1909.11063 [INSPIRE].

[27] A. Bedroya, R. Brandenberger, M. Loverde and C. Vafa, Trans-Planckian Censorship and Inflationary Cosmology, Phys. Rev. D 101 (2020) 103502 [arXiv:1909.11106] [INSPIRE].

[28] L. Aalsma and G. Shiu, Chaos and complementarity in de Sitter space, JHEP 05 (2020) 152 [arXiv: 2002.01326] [INSPIRE].

[29] A. Berera, S. Brahma and J.R. Calderón, Role of trans-Planckian modes in cosmology, arXiv:2003.07184 [INSPIRE].

[30] S.K. Garg and C. Krishnan, Bounds on Slow Roll and the de Sitter Swampland, JHEP 11 (2019) 075 [arXiv: 1807.05193] [INSPIRE].

[31] PLANCK collaboration, Planck 2018 results. X. Constraints on inflation, arXiv:1807.06211 [INSPIRE].

[32] M. Bastero-Gil, A. Berera, R.O. Ramos and J.G. Rosa, Warm Little Inflaton, Phys. Rev. Lett. 117 (2016) 151301 [arXiv: 1604.08838] [INSPIRE].

[33] A. Berera, J. Mabillard, M. Pieroni and R.O. Ramos, Identifying Universality in Warm Inflation, JCAP 07 (2018) 021 [arXiv: 1803.04982] [INSPIRE].

[34] I.G. Moss and C. Xiong, On the consistency of warm inflation, JCAP 11 (2008) 023 [arXiv: 0808.0261] [INSPIRE].

[35] S. del Campo, R. Herrera, D. Pavón and J.R. Villanueva, On the consistency of warm inflation in the presence of viscosity, JCAP 08 (2010) 002 [arXiv: 1007.0103] [INSPIRE].

[36] M. Bastero-Gil, A. Berera, R. Cerezo, R.O. Ramos and G.S. Vicente, Stability analysis for the background equations for inflation with dissipation and in a viscous radiation bath, JCAP 11 (2012) 042 [arXiv: 1209.0712] [INSPIRE].

[37] M. Benetti and R.O. Ramos, Warm inflation dissipative effects: predictions and constraints from the Planck data, Phys. Rev. D 95 (2017) 023517 [arXiv: 1610.08758] [InSPIRE].

[38] M. Bastero-Gil, A. Berera, R.O. Ramos and J.G. Rosa, Towards a reliable effective field theory of inflation, arXiv:1907.13410 [INSPIRE].

[39] S. Bartrum, M. Bastero-Gil, A. Berera, R. Cerezo, R.O. Ramos and J.G. Rosa, The importance of being warm (during inflation), Phys. Lett. B $\mathbf{7 3 2}$ (2014) 116 [arXiv: 1307.5868] [INSPIRE].

[40] K.V. Berghaus, P.W. Graham and D.E. Kaplan, Minimal Warm Inflation, JCAP 03 (2020) 034 [arXiv: 1910.07525] [INSPIRE].

[41] G.B.F. Lima and R.O. Ramos, Unified early and late Universe cosmology through dissipative effects in steep quintessential inflation potential models, Phys. Rev. D 100 (2019) 123529 [arXiv: 1910.05185] [INSPIRE]. 